\title{
Polynomial Degree
}

National Cancer Institute

\section{Source}

National Cancer Institute. Polynomial Degree. NCI Thesaurus. Code C48924.

The highest power of any term in a polynomial. 